\title{
Pneumokokken-Impfung für Erwachsene unter 60 Jahren? Ja
}

Mathias W. Pletz

Die ambulant erworbene Pneumonie (CAP) ist die häufigste Infektion, die in Deutschland zu einer Hospitalisierung führt, und Pneumokokken sind ihr mit Abstand häufigster Erreger. Sie verursachen zwischen 12-85\% aller CAP-Fälle in Europa [9]. Auch in Deutschland sind Pneumokokken mit 30\% der häufigste CAP-Erreger [7].

Die deutschlandweite externe Qualitätssicherung für „Ambulant erworbene Pneumonie“ zeigte, dass 2006 7,5\% der hospitalisierten CAP-Patienten zwischen50 und59Jahrealt waren ( $n=15094)$. Die Letalität steigt nach dem 40 . Lebensjahr erstmalig signifikant an und nimmt danach kontinuierlich zu. Bei den 50-59-Jährigen beträgt sie bereits 7,5\% [3]. Dies demonstriert eindrucksvoll, dass Pneumokokken-Infektionen auch bei Erwachsenen unter 60 relevant sind.

Die Impfung jüngerer, (noch) nicht immunoseneszenter Menschen induziert eine stärkere und nachhaltigere Impfantwort [6]. Dies gilt auch für die Impfung mit der 13-valenten Konjugatvakzine (PCV13): 50-59-Jährige zeigten signifikant höhere Antikörperspiegel gegen 9 der 13 Serotypen als 60-64-Jährige [10]. Aber hält die Wirkung auch an? In einer Studie zu invasiven Pneumokokken-Infektionen nahm die Schutzwirkung bereits 2 Jahre nach Impfung mit der 23-valenten Polysaccharidvakzine (PSV23) ab [1]. Für PCV13 zeigte aber die CAPITA-Studie auch nach einer Beobachtungszeit von 4 Jahren eine bleibende Schutzwirkung [2].

Was spricht gegen eine generelle Impfung schon ab dem 50. Lebensjahr? Da beide Pneumokokkenvakzinen (PCV13 und PSV23) gut verträglich sind und schwere Nebenwirkungen extrem selten auftreten, spielt die Sicherheit bei der Nutzen-RisikoAnalyse eine nachgeordnete Rolle. Jährlich werden ca. 15000 Patienten zwischen 50 und 59 Jahren aufgrund einer CAP hospitalisiert [3]. Dabei liegt die Pneumokokkenrate bei 12-85\% [9], 64\% davon sind die PCV13-Serotypen (CAPNETZ, nicht publizierte Daten). Bei einer PCV13-Effektivität von $45 \%$ [2] könnten durch eine generelle Impfung mit PCV13 ab 50 jährlich zwischen 521 und 3695 Hospitalisierungen und 39-77 Todesfälle in der Altersgruppe der 50-59-Jährigen verhindert werden. Diese Zahlen liegen in der Größenordnung der Krankheitslast von Meningokokken der Serogruppe C vor Einführung der entsprechenden Standardimpfung bei Kleinkindern in 2006. Wie viele am- bulante Fälle verhindert werden könnten, ist unklar. Aber Daten aus Deutschland legen nahe, dass die Inzidenz der ambulant behandelten Pneumonien bis zu 2-3mal höher ist als die der stationären CAP-Fälle [3, 8].

Da Menschen zwischen 50 und 59 Jahren in der Regel im Berufsleben stehen, verursachen Pneumonien in dieser Altersgruppe nicht nur Kosten der medizinischen Versorgung, sondern auch einen Ausfall an Produktivität, was bei der KostenNutzen-Analyse betrachtet werden sollte [4]. Eine Modellierung der CAPITA-Daten zeigte für die Niederlande, dass eine Standard-Impfung ab dem 50. Lebensjahr mit PCV13 kosteneffektiv ist [5].

Zusammenfassend ist das Risiko einer Pneumokokken-Pneumonie bei 50-59-Jährigen zwar geringer als bei älteren Personen, die Krankheitslast ist aber dennoch beträchtlich. Für eine generelle Impfung bereits ab 50 statt ab 60 spricht auch die in dieser Lebensdekade signifikant bessere Immunogenität. Nachteile der früheren Impfung (z.B. reduzierte Schutzwirkung im höheren Alter) sind für PSV23 zu erwarten. Die Schutzwirkung von PCV13 war über die gesamte Beobachtungsdauer der CAPITA-Studie erhalten. Des Weiteren ist eine wiederholte Impfung mit PCV13 im Gegensatz zu einer - nicht mehr generell empfohlenen Wiederholungsimpfung mit PSV23 nicht mit einem verminderten Ansprechen (Hyporesponsiveness) assoziiert. Folglich besteht die Möglichkeit einer Wiederholungsimpfung im höheren Alter.

\section{Fazit}

- Für eine Impfung gegen Pneumokokken bereits ab 50 sprechen die bessere Immunogenität bei unter 60 -Jährigen und die Krankheitslast der Pneumokokken-Pneumonie: Morbidität und Mortalität steigen bereits vor dem 50. Lebensjahr an. Eine solche Empfehlung wäre aber nur für PCV13 sinnvoll, da diese im Gegensatz zu PSV23

> gegen die häufige nicht-bakteriämische Pneumokokken-Pneumonie schützt [7] und

> eine längere Schutzdauer als PSV23 induziert. Zudem ist aufgrund fehlender Hyporesponsiveness auch eine Wiederholungsimpfung im höheren Alter prinzipiell möglich.

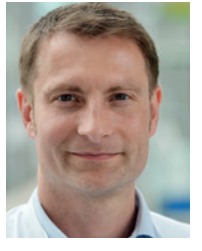

Prof. Dr. Mathias W. Pletz ist Direktor des Zentrums für Infektionsmedizin und Krankenhaushygiene und leitet die BMBF-Forschergruppe "Klinische Infektiologie - neue Strategien gegen multiresistente Erreger" am Universitätsklinikum Jena mathias.pletz@ med.uni-jena.de

Literaturverzeichnis unter http://dx.doi. org/10.1055/s-0041-107750

\section{Interessenkonflikt}

Der Autor gibt an, Honorare für Vorträge und Beratertätigkeiten von Pfizer, GSK und MSD sowie Unterstützung von Forschungsprojekten durch Pfizer erhalten zu haben.

DOI 10.1055/s-0041-107750 Published online 3.11.2015 Dtsch Med Wochenschr 2015; 140: 1775

(c) Georg Thieme Verlag KG . Stuttgart · New York . ISSN 0012-0472 


\section{Literatur}

1 Andrews NJ, Waight PA, George RC et al. Impact and effectiveness of 23-valent pneumococcal polysaccharide vaccine against invasive pneumococcal disease in the elderly in England and Wales. Vaccine 2012; 30: 6802-6808

2 Bonten M], Huijts SM, Bolkenbaas M et al. Polysaccharide conjugate vaccine against pneumococcal pneumonia in adults. N Engl ] Med 2015; 372: 1114-1125

3 Ewig S, Birkner N, Strauss R et al. New perspectives on community-acquired pneumonia in 388406 patients. Results from a nationwide mandatory performance measurement programme in healthcare quality. Thorax 2009; 64: 1062-1069

4 Kuhlmann A, Theidel U, Pletz MW et al. Potential cost-effectiveness and benefit-cost ratios of adult pneumococcal vaccination in Germany. Health Econ Rev 2012; 2: 4

5 Mangen MJ, Rozenbaum MH, Huijts SM et al. Cost-effectiveness of adult pneumococcal conjugate vaccination in the Netherlands. Eur Respir ] 2015; DOI: 10.1183/13993003.003252015

6 Oviedo-Orta E, Li CK, Rappuoli R. Perspectives on vaccine development for the elderly. Curr Opin Immunol 2013; 25: 529-534
7 Pletz MW, von Baum $\mathrm{H}$, van der Linden $\mathrm{M}$ et al. The burden of pneumococcal pneumonia - experience of the German competence network CAPNETZ. Pneumologie 2012; 66: 470-475

8 Schnoor M, Hedicke J, Dalhoff K et al. Approaches to estimate the population-based incidence of community acquired pneumonia. J Infect 2007; 55: 233-239

9 Torres A, Blasi F, Peetermans WE et al. The aetiology and antibiotic management of community-acquired pneumonia in adults in Europe: a literature review. Eur ] Clin Microbiol Infect Dis 2014; 33: 1065-1079

10 Pfizer. Fachinformation zu Prevenar 13 Injektionssuspension https://www.pfizermed.de/fileadmin/ produktdatenbank/pdf/012126_freigabe.pdf Letzter Zugriff am 26.10.15 